
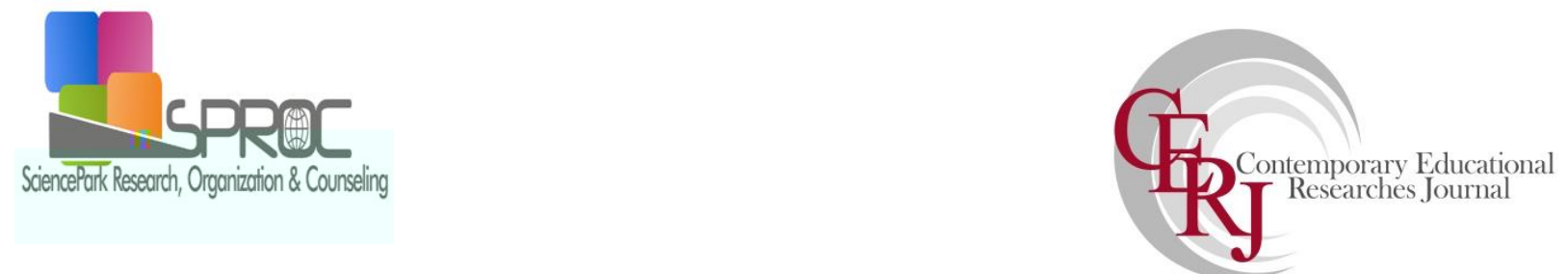

www.cerj.eu

\title{
An analysis of speech disfluencies of Turkish speakers: Influence of educational background
}

Ayse Altiparmak ${ }^{*}$

Gulmira Kuruoglu

Suggested Citation:

Contemporary Educational Researches Journal 7

Abstract

Ayse Altiparmak,

E-mail address 
Contemporary Educational Researches Journal 7

1. Introduction

2. Method

2.1. Participants

2.2. Procedure for collecting and analyzing the speech data 


\section{Data analysis and findings}

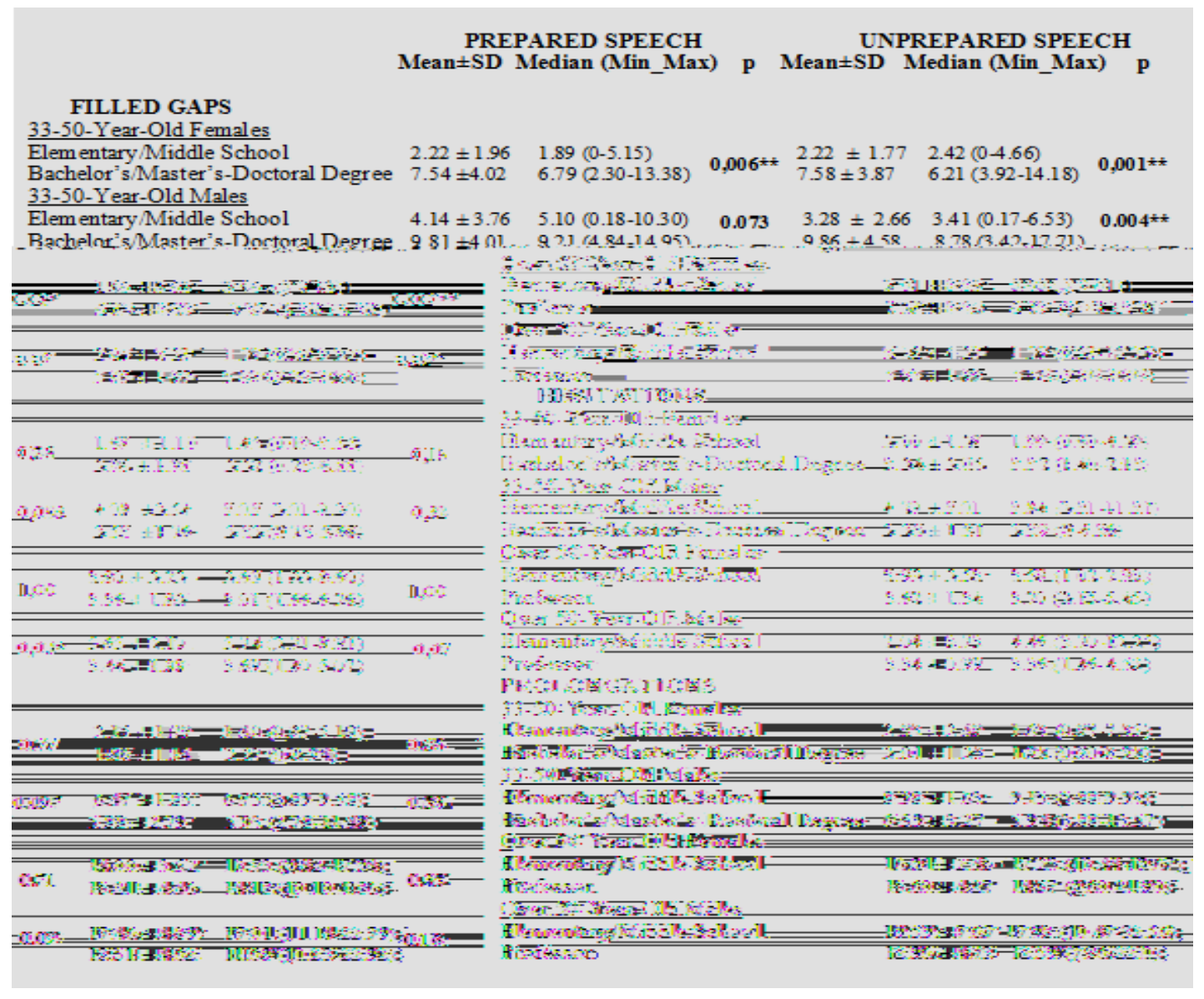


FALSE STARTS

33-50-Year-Old Females

Elementary/Middle School

Bachelor's/Master's-Doctoral Degree

33-50-Year-Old Males

Elementary/Middle School

Bachelor's/Master's-Doctoral Degree

Over 50-Year-Old Females

Elementary/Middle School

Professor

Over 50-Year-Old Males

Elementary/Middle School

Professor

SLIPS OF THE TONGUE

33-50-Year-Old Femal es

Elementary/Middle School

Bachelor's/Master's-Doctoral Degree

33-50-Year-Old Males

Elementary/Middle School

Bachelor's/Master's-Doctoral Degree

Over 50-Year-Old Females

Elementary/Middle School

Professor

Over 50-Year-Old Males

Elementary/Middle School

Professor

REPETITIONS

33-50-Year-Old Females

Elementary/Middle School

Bachelor's/Master's-Doctoral Degree

33-50-Year-Old Males

Elementary/Middle School

Bachelor's/Master's-Doctoral Degree

Over 50-Year-Old Females

Elementary/Middle School

Professor

Over 50-Year-Old Males

Elementary/Middle School

Professor
PREPARED SPEECH

Mean \pm SD Median (Min.Max)

$0.45 \pm 0.40 \quad 0.33(0-1.25)$

$0.86 \pm 0.65 \quad 0.88(0-1.68)$

$2.30=1.83 \quad 1.68(0.51-5.04)$

$0.89=0.24 \quad 0.85(0.53-1.25)$

$0.79=0.47 \quad 0.96(0.20-1.47)$

$1.02=0.33 \quad 0.92(0.61-1.49)$

$1.20=0.73 \quad 0.97(0.52-2.45)$

$1.02=0.52 \quad 0.77(0.44-1.73)$

$0.36 \pm 0.30 \quad 0.38(0-0.83)$

$1.19=0.19$

$0.73=0.30$

$0.36 \pm 0.28$

$0.31 \pm 0.26$

$0.27 \pm 0.18$

$0.63=0.45$

$0.46 \pm 0.39$

$0.09(0-0.51)$

$0.69(0.34-1.11)$

$0.35(0-0.92)$

$0.27(0-0.61)$

$0.33(0-0.50)$

$0.84(0-1.13)$

$0.44(0.11-1.24)$

$0.23 \pm 0.19 \quad 0.25(0-0.60)$

$0.15=0.15 \quad 0.08(0-0.39)$

$0.51 \pm 0.81 \quad 0.21(0-2.22)$

$0.10=0.18 \quad 0(0-0.48)$

$0.14=0.18 \quad 0(0-0.40)$

$0.31=0.35 \quad 0.22(0-1.01)$

$0.32=0.46 \quad 0.16(0-1.26)$

$0.34 \pm 0.35 \quad 0.24(0-0.87)$
0.23

$0.39=0.35 \quad 0.34(0-1.09)$

$0.40 \pm 0.33 \quad 0.42(0-0.97)$

$0.16 \quad 1.81=0.96 \quad 1.23(0.90-3.37)$

$0.82=0.35 \quad 0.90(0.17-1.14)$

$0.53 \quad 0.53=0.39 \quad 0.49(0-1.24)$

$1.26=0.49 \quad 1.19(0.51-1.96)$

$0.86=0.58 \quad 1.04(0-1.55)$

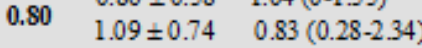

$\mathbf{0 , 3 3} \quad 0.21=0.16 \quad 0.25(0-0.39) \quad \mathbf{0 , 7 7}$

$0.18 \pm 0.19 \quad 0.23(0-0.49)$

$0.03 * \quad 0.52=0.24 \quad 0.61(0.14-0.73)$

$0,03 * \quad 0.29=0.22 \quad 0.24(0-0.67)$

$0, \$ 0 \quad 0.51=0.33 \quad 0.58(0-0.98)$

$0.18 \pm 0.16 \quad 0.18(0-0.39)$

$0.53 \quad 0.52=0.30 \quad 0.34(0.27-1.03)$

$0,53 \quad 0.44=0.20 \quad 0.38(0.25-0.84)$

$0,61 \quad 0.02 \pm 0.05 \quad 0(0-0.12)$

$0.02=0.04 \quad 0.01(0-0.12)$

$0.48=0.65 \quad 0.33(0-1.84)$

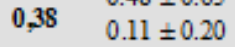

$0(0-0.46)$

$0,38 \quad 0.35=0.45$

$0.20 \pm 0.02$

$0.24(0-1.23)$

$0(0-0.05)$

$0.80 \quad 0.14=0.11 \quad 0.16(0-0.27)$

$0, \$ 0 \quad 0.32 \pm 0.37 \quad 0.15(0-1.05)$
0.01 *

0.71

0,21

1,00

0.86

0.01 *

0.053

0,46

0,26

0,45

0,71 
4. Discussion and conclusions 
Contemporary Educational Researches Journal 7

-

$\bullet$ 
Contemporary Educational Researches Journal 7

112 


\section{References}

Revista de Logopedia Foniatría y Audiología 31

Speech, Language and Hearing Research 41,

Journal of

Proceedings of ICSLP 2000

Language and Speech 44

Proceedings of the 14th International Conference of Phonetic Sciences

Cambridge grammar of English: a comprehensive guide

Psychology 70

Journal of Personality and Social Cognitive Psychology, 37

Language and

Cognitive Processes 5,

The Rice University

Studies 66

Language and Speech 24

Journal of Communication Disorders, 31,

Learning and Memory 20

The Neurocognition of Language

Technology

Proceedings of the European Conference on Speech Communication and

Conference on Spoken Language Processing (ICSLP) '92

Language., 9,

Proceedings of DiSD '03

Language and Speech 33

Unpublished Doctoral Dissertation

Language Processing

Proceedings of International Conference on Spoken

Memory and Cognition 14,

Linguistics 16

International Journal of Corpus 